\title{
Corrigendum to 'Growth inhibition properties of the putative prostate cancer biomarkers PSP94 and CRISP-3' by Aleyde van Eynde et al.
}

\author{
Aleyde van Eynde, Kirill Litovkin and Mathieu Bollen
}

Asian Journal of Andrology (2011) 13, 348; doi:10.1038/aja.2010.175

Correction to: Asian Journal of Andrology 2010. doi: 10.1038/ aja.2010.120. Advance online publication (22 November 2010)

In this article, published online 22 November 2010 and in this issue, the name of the first author, listed as Bhakti RP, in Reference 6 was incorrect; the correct name should be Pathak BP.

There is a misspelling in the penultimate paragraph of the text. A1GB should have been spelled A1BG so that the sentence should read: "In human blood, however, CRISP-3 is known to bind to the human plasma protein A1BG...".

Finally, in the figure there are two misspellings: CRIPS-9 should be CRISP-9 and 37LRD should be 37LRP. The correct figure is provided here.

We offer our profound apologies to the readers for any confusion that arises due to our careless errors. 